\title{
Differences in Automatic Thoughts Presented During Sexual Activity Between Sexually Functional and Dysfunctional Men and Women
}

\author{
Pedro J. Nobre · José Pinto-Gouveia
}

Published online: 31 October 2007

(C) Springer Science+Business Media, LLC 2007

\begin{abstract}
The study is aimed at investigating the differences between individuals with and without sexual dysfunction on the automatic thoughts content (reported as usually presented) during sexual activity. A total of 491 individuals (163 women and 232 men without sexual problems and 47 women and 49 men with a DSM-IV diagnosis of sexual dysfunction) answered the Sexual Modes Questionnaire (SMQ; Nobre and Pinto-Gouveia (J Sex Res 40:368-382, 2003). Results indicated that men and women with sexual dysfunction reported having had significantly more negative thoughts during sexual activity compared to sexually healthy individuals. Failure and disengagement thoughts (I'm not satisfying my partner, I'm not getting turned on, when will this be over?), sexual abuse thoughts (this is disgusting, he only wants to satisfy himself), and lack of erotic thoughts were significantly more common in women with sexual dysfunction compared to sexually healthy women. Additionally, men with sexual dysfunction presented significantly higher scores on erection concern thoughts (I must be able to have intercourse, I must achieve an erection), failure anticipation thoughts (this is not going anywhere, I'm condemned to failure), and lack of erotic thoughts compared to men without sexual dysfunction. Overall, findings support clinical observations and experimental findings, indicating that cognitive distraction from erotic cues is strongly associated with sexual dysfunction. The increased use of cognitive techniques on the treatment of sexual dysfunction is a major implication of the study.
\end{abstract}

Keywords Automatic thoughts $\cdot$ Cognitive distraction $\cdot$ Sexual dysfunction · Assessment

\footnotetext{
P. J. Nobre (ه)

Departamento de Educação e Psicologia, Universidade de Trás-os-Montes e Alto Douro,

Rua Amorim de Carvalho, 97, Senhora da Hora 4460, Portugal

e-mail: pedro.j.nobre@clix.pt; pnobre5@gmail.com

J. Pinto-Gouveia

Faculdade de Psicologia e Ciências da Educação da Universidade de Coimbra, Coimbra, Portugal
} 


\section{Introduction}

The study of automatic thoughts constitutes one of the most common areas of research in the field of cognitive therapy. In fact, this dimension assumes the role of the most easily observable variable within the cognitive system. According to Beck (1967), automatic thoughts are images or cognitions presented by subjects as a result of the cognitive schema or core belief that is activated in a particular moment. Thus, automatic thoughts reflect the content of the more central and tacit structures of the cognitive system. These images and thoughts reflect the meaning subject's are assigning to a given situation and are deeply associated with the emotional and behavioral response to that event. Moreover, automatic thoughts play an important role in the therapeutic process, being used as a preferential access pathway to the cognitive system and helping to understand the core beliefs underlying the several psychological disorders (Beck 1995). Therefore, it is comprehensible that different theoretical conceptualizations of psychopathological phenomena have been characterized according to the automatic thoughts that are most commonly associated with them. Beck (1996) suggested that specific phobia is characterized by thoughts about specific dangers, general anxiety disorders by thoughts about general and mid- to long-term dangers, whereas depression is characterized by thoughts about loss.

Regarding the sex field, several empirical studies have emphasized the role of cognitive variables on sexual functioning. Research on cognitive distraction, attentional focus, and performance demand thoughts has been conducted in the last two decades.

Geer and Fuhr (1976) developed an experimental design conceived to investigate the sexual response of sexually healthy individuals during simultaneous exposure to two types of different audio stimuli. Results have shown that the increasing complexity of the arithmetic tasks presented to the subjects interfered with their erection levels, which became progressively lower. This negative influence of cognitive distraction on sexual functioning was further confirmed by several studies using men and women without sexual dysfunction (Adams et al. 1985; Beck et al. 1987; Elliot and O'Donohue 1997; Farkas et al. 1979; Viglietta 1982). However, when extending this experimental design to samples of men with sexual dysfunction, findings were different. Abrahamson et al. (1985) indicated that, contrary to sexually healthy men, individuals with erectile disorders did not present a decrease in their erection levels when performing a distractive task of cognitive nature during exposure to erotic materials. The authors suggested that men with sexual dysfunction were already focused on non-erotic stimuli (performance related concerns). Thus, results indicated transference of attentional focus from a non-erotic cue (performance related concerns) to another non-erotic cue (cognitive task), with no implications on sexual response.

In an interesting research design, Abrahamson et al. (1989) tested this hypothesis, studying the differential effect of a neutral (nonsexual) distractor (comparing the length of a line with the length of a line viewed earlier) and a sexual distractor (estimating their levels of erectile response and its sufficiency for intercourse) on sexual response (penile circumference change) presented by men with and without sexual dysfunction during exposure to erotica. Results indicated that, whereas sexually healthy men presented higher erectile responses during the sexual distraction task, men with sexual difficulties presented a reverse response pattern. These findings indicate that the erectile response of men with sexual dysfunction is more affected by cognitive distractions related to sexual performance (potentially replicating the natural context where the sexual difficulties take place) than by neutral distractors. These data seem to corroborate the idea that during sexual activity, men with sexual problems focus their attention on cues related to erection and sexual performance, which negatively interfere with sexual response. 
Based on these studies, Barlow (1986) developed a cognitive-affective model of sexual dysfunction. In this model it is suggested that, whereas men without sexual problems, when exposed to sexual events, focus their attention on erotic cues, men with sexual dysfunction focus their attention on negative thoughts regarding public consequences of not performing or other non-erotic issues. Several sex therapists have corroborated this conceptual model, suggesting that during sexual activity, men with sexual difficulties tend to present thoughts related to performance concerns (erection and intercourse concerns) and failure anticipation and its consequences (Carey et al. 1993; Hawton 1985, 1989; Rosen et al. 1994; Wincze and Barlow 1997; Zilbergeld 1999).

Besides laboratorial studies and clinical suggestions, research on the specific content of thoughts presented during sexual activity have been scarce. Moreover, the majority of the studies were conducted with men and focused mainly on issues regarding erectile response and performance demands. One exception is Dove and Wiederman's study (2000). The authors developed a questionnaire to assess the attentional focus of women during sexual activity (attentional focus on body appearance or attentional focus on sexual performance), and its influence on sexual functioning and satisfaction. Results indicated that women with higher levels of cognitive distraction (attentional focus on body appearance or sexual performance, rather than on erotic cues) present lower sexual satisfaction and less frequent orgasms during sexual activity.

These data partially corroborate findings from studies conducted in men, suggesting that attentional focus on non-erotic (off-task) cues has a negative interference on sexual response. It seems, however, that the specific content of the distractive attentional focus differentiates men and women. While in men the privileged focus is on erectile response (or the lack of), in women the focus seems to be primarily on self body image (or stimuli indicating low body appearance).

The aim of the present study is to assess the differences between men and women with and without sexual dysfunction regarding the content of the thoughts reported as usually presented during sexual activity. The study is based on questionnaires (automatic thought sub-scale of the Sexual Modes Questionnaires-SMQ; Nobre and Pinto-Gouveia 2003) containing a group of thoughts regarding a diverse range of issues that include erotic/sexual thoughts and negative cognitions representing off-task thoughts. Overall, and according to Barlow's model (1986), it is expected that sexually healthy individuals focus their attention on sexual and erotic stimuli (higher frequency of erotic thoughts), whereas individuals with sexual dysfunction focus their attention on non-sexual stimuli (higher frequency of performance related thoughts, and thoughts related to the possible negative consequences of a sexual failure). Specifically, we expect to find significantly higher cognitions related to failure anticipation and erection concerns in men with sexual dysfunction, compared to sexually healthy men. In women, we expect to find higher sexual abuse thoughts, failure and disengagement thoughts, partners lack of affection, passivity and control thoughts, and low self body image thoughts in the clinical group, compared to the non-clinical.

\section{Method}

Participants and Procedures

A total of 491 individuals ( 210 women and 281 men) participated in the study. A control sample of 395 individuals (163 women and $232 \mathrm{men}$ ) and a clinical sample of 96 individuals (47 women and 49 men) were constituted. 
Participants from the control sample were recruited in different regions of Portugal by a group of volunteer students from Universidade de Trás-os-Montes e Alto Douro. This group of students collected the sample in their hometowns throughout the country using non-random methods (convenience sample). Participants were contacted door to door by the volunteers who explained the purpose of the study and gave them the questionnaire with the instructions. Students were instructed to avoid asking people they knew. Participants who accepted to answer the questionnaires signed a consent statement form approved by the IRB. In order to assure anonymity, individuals were instructed to answer the questionnaires when alone and in the privacy of their homes and then to return them by mail using pre-stamped envelopes. Participants were not paid for their participation.

In order to control the presence of sexual dysfunction, participants who presented scores lower than the cutoffs on the different dimensions assessed by the International Index of Erectile Function (IIEF, Rosen et al. 1997) and the Female Sexual Function Index (FSFI, Rosen et al. 2000)] were excluded. The cutoff scores used were the following: for the men, sexual desire $=5.2$, erectile function $=22$, orgasmic function $=5.9$, intercourse satisfaction $=7.7$, and overall satisfaction $=6.9$; for the women, sexual desire $=3.0$, sexual arousal $=4.1$, lubrication $=4.6$, orgasm $=3.8$, pain $=4.4$, and sexual satisfaction $=3.9$. With the exception of erectile function, where the optimal cutoff scores were calculated and published (Cappelleri et al. 1999), the remaining cutoff scores were calculated by subtracting one Standard Deviation from the average on the different domains of the IIEF (Rosen et al. 1997) and FSFI (Rosen et al. 2000) from samples of subjects without sexual dysfunction (means and SD presented by Rosen et al. 1997, 2000). Using this criterion, 71 men and 103 women from the total community sample initially gathered, were excluded from the study. The control sample was collected between September 2000 and April 2002 and the response rate was $30.6 \%$ (the demographic characteristics of the control sample are shown in Table 1).

Participants from the clinical sample were recruited among the patients of the sexology clinic of Coimbra's University Hospital (an outpatient clinic of a central hospital serving the population of Coimbra and its region). Participants diagnosed with sexual dysfunction, using the Diagnostic and Statistical Manual of Mental Disorders (DSM-IV; American Psychiatric Association 1994) criteria, constituted this clinical group. The principal diagnoses assigned were the following: for the men, erectile disorder (69.4\%), premature ejaculation $(22.4 \%)$, orgasmic disorder (4.1\%), hypoactive sexual desire (2\%), and sexual dysfunction not otherwise specified (2\%); for the women, hypoactive sexual desire (40.4\%), vaginismus (25.5\%), orgasmic disorders $(21.3 \%)$, dyspareunia (6.4\%), sexual arousal $(4.3 \%)$, and sexual aversion $(2.1 \%)$. A total of 21 women (43\%), and 9 men (18\%) presented additional clinical diagnoses (secondary diagnosis). After completing a clinical assessment (conducted by a group of trained sex therapists from the clinic using an unpublished structured interview for sexual dysfunction), patients who were assigned with a DSM-IV diagnosis of sexual dysfunction were approached about the study by a member of the research team and given option to decline. An explanation of the purpose of the study was given and a consent form was signed. Participants then answered the questionnaire by themselves in a private space and returned them directly to the member of the team. Participants took $40 \mathrm{~min}$ in average to answer the questionnaires. The clinical sample was collected between September 2000 and December 2001, and the response rate was $94.8 \%$ (the demographic characteristics of the clinical sample are shown in Table 1). Statistically significant differences were observed between clinical and control groups in the demographic variables of age (male sample: $t(277)=3.15, p<.01$ ), marital status (male sample: $\chi^{2}(5$, $N=281)=12.44, p<.05$; female sample: $\left.\chi^{2}(5, N=207)=18.10, p<.01\right)$, and education (male sample: $\chi^{2}(6, N=280)=117.56, p<.001$; female sample: $\chi^{2}(6, N=206)=14.11$, $p<.05)$. No differences were found for age in the female sample $[t(208)=-1.01, p=.32]$. 
Table 1 Demographic characteristics of the clinical and control groups

\begin{tabular}{|c|c|c|c|c|}
\hline & \multicolumn{2}{|l|}{ Control group } & \multicolumn{2}{|l|}{ Clinical group } \\
\hline & Female $(n=163)$ & Male $(n=232)$ & Female $(n=47)$ & Male $(n=49)$ \\
\hline \multicolumn{5}{|l|}{ Age } \\
\hline M & 30.41 & 35.78 & 28.7 & 43.0 \\
\hline Min-Max & $18-75$ & $18-79$ & $19-50$ & $18-67$ \\
\hline SD & 11.38 & 14.27 & 6.7 & 14.4 \\
\hline \multicolumn{5}{|l|}{ Marital status } \\
\hline Single & $57.5 \%$ & $40.9 \%$ & $23.4 \%$ & $26.5 \%$ \\
\hline Married & $33.8 \%$ & $53.0 \%$ & $63.8 \%$ & $61.2 \%$ \\
\hline Divorced & $3.8 \%$ & $3.4 \%$ & $4.3 \%$ & $2.0 \%$ \\
\hline Widowed & $0.6 \%$ & $0.4 \%$ & $0.0 \%$ & $0.0 \%$ \\
\hline Living together & $3.1 \%$ & $1.7 \%$ & $6.4 \%$ & $10.2 \%$ \\
\hline Separated & $1.3 \%$ & $0.4 \%$ & $2.1 \%$ & $0.0 \%$ \\
\hline \multicolumn{5}{|l|}{ Educational level } \\
\hline 0 years of education & $0.6 \%$ & $0.0 \%$ & $0.0 \%$ & $0.0 \%$ \\
\hline$<4$ years & $0.0 \%$ & $0.4 \%$ & $0.0 \%$ & $0.0 \%$ \\
\hline 4 years & $3.1 \%$ & $3.9 \%$ & $10.6 \%$ & $38.8 \%$ \\
\hline $5-6$ years & $3.1 \%$ & $2.2 \%$ & $10.6 \%$ & $24.5 \%$ \\
\hline $7-9$ years & $3.8 \%$ & $5.6 \%$ & $6.4 \%$ & $12.2 \%$ \\
\hline $10-12$ years & $24.5 \%$ & $18.2 \%$ & $31.9 \%$ & $22.4 \%$ \\
\hline$>12$ years & $64.7 \%$ & $69.7 \%$ & $40.4 \%$ & $2.0 \%$ \\
\hline
\end{tabular}

\section{Materials}

\section{The International Index of Erectile Function (IIEF)}

The IIEF (Rosen et al. 1997) is a 15-item, brief, self-administered measure assessing different areas of sexual functioning in men. A principal component analysis identified five factors: erectile function, orgasmic function, sexual desire, intercourse satisfaction and overall satisfaction. Psychometric studies supported the validity (significant mean score differences between a clinical and a control group) and reliability (Cronbach's $\alpha=.73$ and higher, and test-retest from $r=.64$ to .84 ) of the measure. Studies with clinical samples demonstrated its sensitivity and specificity for detecting treatment related changes (Rosen et al. 1997). The measure allows the calculation of specific indexes for each dimension as well as a sexual function total index (calculated through the sum of the specific dimensional indexes), with higher scores indicating greater levels of sexual functioning (sexual desire: 2-10, erectile function: 1-30, orgasmic function: $0-10$, intercourse satisfaction: $0-15$, overall satisfaction: $2-10$, total: $5-75$ ).

\section{The Female Sexual Function Index (FSFI)}

The FSFI (Rosen et al. 2000) is a 19-item instrument, easily administered and scored, providing detailed information on the major dimensions of sexual function. A principal component analysis identified six factors: sexual interest/desire, sexual arousal, lubrication, orgasm, sexual satisfaction and sexual pain. The measure presents acceptable test-retest 
reliability ( $r=.79-.86$ ), internal consistency (Cronbach's $\alpha=.82$ and higher), and validity (demonstrated by significant mean difference scores between a clinical and a control group) (Rosen et al. 2000). The measure allows the calculation of specific indexes for each dimension as well a sexual function index (calculated through the sum of the specific dimensional indexes), with higher scores indicating greater levels of sexual functioning (desire: 1.2-6, arousal: $0-6$, lubrication: $0-6$, orgasm: $0-6$, global satisfaction: $0.8-6$, pain: $0-6$, total, 2-36).

\section{Sexual Modes Questionnaire (SMQ)}

The SMQ (Nobre and Pinto-Gouveia 2003) is a measure aimed at assessing thoughts, emotions and sexual response reported has usually presented during sexual activity. The questionnaire is composed of three sub-scales: Automatic thought sub-scale (AT), Emotional response subscale (ER), and Sexual response sub-scale (SR).

The automatic thought sub-scale (AT) has a male and a female version. The male version is composed of 30 items and the female version of 33 items that evaluate thoughts and images reported as usually presented by individuals during sexual activity. The participants are asked to rate the frequency (from 1-never to 5-always) in which they usually experience those thoughts during sexual activity. Thoughts included in the scale were selected based on its theoretical and clinical relevance, assessing whether the thought content is oriented to sexual/ erotic stimuli or to non-relevant cues.

Psychometric studies have supported the reliability and validity of the sub-scales (Nobre and Pinto-Gouveia 2003). Test-retest data indicated a statistically significant correlation $(r=.95, p<.01)$ for the total scale between two consecutive administrations of the female version, and a moderate correlation $(r=.65, p<.05)$ for the male version (possibly due to the low sample size, $n=9$ ). Cronbach's $\alpha=.88$ for the male and .87 for the female version supported the high internal consistency of both scales. Convergent Validity indicated that the AT sub-scales are strongly associated with measures of sexual functioning. The female version is significantly correlated with the Female Sexual Function Index $(r=.49, p<.01)$, and the male version with the International Index of Erectile Function $(r=.60, p<.05)$.

Both versions of the automatic thought sub-scale were submitted to factor analysis (Nobre and Pinto-Gouveia 2003). A principal component analysis with varimax rotation of the female version identified 6 factors accounting for $53.1 \%$ of the total variance:

1. Sexual abuse thoughts: the dimension, which expresses thoughts of being abused, disrespected and even violated by the sexual partner.

2. Failure and disengagement thoughts: the domain that is constituted by thoughts of incapacity for sexual performance, and lack of motivation to engage in sexual activity.

3. Partner's lack of affection: the factor characterized by thoughts of not being treated with care and affection by the partner during sexual activity. This dimension reflects the dichotomy between sex as an affective activity and sex as a physical activity.

4. Sexual passivity and control: the dimension represented by thoughts of female sexual passivity and control, reflecting the idea that women must wait for the men's first step in order to match cultural values (not being seen as frivolous) and also in order to prevent eventual emotional harm.

5. Erotic thoughts: the domain formed by thoughts of sexual erotic content. Since these items were scored in reverse order, higher results on this factor represent lack of erotic thoughts content during sexual activity.

6. Low self body-image thoughts: the factor characterized by thoughts of not being comfortable with one's body image. 
In the male version, the principal component analysis identified 5 factors that accounted for $54.7 \%$ of the total variance:

1. Failure anticipation and catastrophyzing thoughts: the factor characterized by thoughts of incapacity to perform, failure anticipation and magnifying the negative consequence of failure.

2. Erection concern thoughts: the dimension constituted by thoughts specifically related to penis reaction and concerns about the capacity for intercourse.

3. Age and body function related thoughts: the domain represented by thoughts of being old and its implications regarding sexual activity and partner's attraction.

4. Negative thoughts toward sex: the factor characterized by conservative and negative thoughts toward sexuality.

5. Erotic thoughts: the dimension represented by sexual and erotic thoughts. Since these items were scored in reverse order, higher values on this domain represent lack of erotic thoughts content during sexual activity.

In both male and female versions specific dimensional scores for each factor and a total score might be calculated, with higher scores representing negative thoughts during sexual activity.

\section{Results}

Automatic Thoughts and Sexual Dysfunction in Women

A multivariate analysis of covariance (MANCOVA) was performed in order to assess the association between thoughts presented during sexual activity and sexual functioning in women. The analysis used sexual functioning ( $1=$ clinical group, $2=$ control group $)$ as the independent variable, and the scores on the six dimensions of the automatic thought sub-scale, as dependent variables. Since the variables age, marital status and educational level showed a statistically significant effect on the dependent variables [age: $F(234,1125)=1.26, p<.01$; marital status: $F(18,626)=3.97, p<.001$; educational level: $F(30,882)=2.10, p<.01$ ], a decision was made to include them as covariates (in order to control their effects). After controlling these effects, the multivariate analysis for sexual functioning (clinical group/ control group) was statistically significant $[F(6,142)=22.61, p<.001]$.

The univariate tests (see Table 2) confirmed the results from the multivariate analysis showing statistically significant effects for sexual functioning on several thought dimensions. Specifically, women with sexual dysfunction presented significantly higher scores $(p<.001)$ on sexual abuse thoughts $\left(\eta^{2}=.11\right)$, failure and disengagement thoughts $\left(\eta^{2}=.43\right)$, lack of erotic thoughts $\left(\eta^{2}=.23\right)$, and on the total automatic thought sub-scale $\left(\eta^{2}=.20\right)$. Regarding the remaining dimensions (partner's lack of affection, sexual passivity/control and low self body image thoughts), the differences did not reach statistical significance.

A discriminant analysis was performed in order to investigate the individual contribution of each thought dimension to distinguish women with and without sexual dysfunction. The six dimensions of the AT sub-scale (matched for age, marital status and educational level) ${ }^{1}$

\footnotetext{
${ }^{1}$ In order to control the effect of the variables: age, marital status and educational level, the discriminant analysis was performed using a sample constituted by the female clinical group $(n=47)$ and a sub-sample of the control group $(n=53)$ matched for age [clinical group $(M=28.66)$, control group $(M=28.87), t(98)=-0.13$, $p=.90]$, marital status $\left[\chi^{2}(4)=2.53, p=.64\right]$ and educational level [Mann-Whitney test for the comparison of the mean orders of the clinical (49.19) and control group (51.66), $z=-.44, p=.66]$.
} 
Table 2 Female thoughts as a function of sexual functioning (clinical group/control group): univariate analysis of covariance (covariables: age, marital status and educational level)

\begin{tabular}{|c|c|c|c|c|c|c|c|}
\hline \multirow[t]{3}{*}{ Thought dimensions } & \multicolumn{4}{|l|}{ Group } & \multirow[t]{3}{*}{$F(1,147)$} & \multirow[t]{3}{*}{$p$} & \multirow[t]{3}{*}{$\eta^{2}$} \\
\hline & \multicolumn{2}{|c|}{ Clinical $(n=36)$} & \multicolumn{2}{|c|}{ Control $(n=116)$} & & & \\
\hline & $M$ & SD & $M$ & SD & & & \\
\hline F1 Sexual abuse thoughts & 13.31 & 4.29 & 9.97 & 2.90 & $17.8 * * *$ & .000 & .11 \\
\hline F2 Failure/disengagement & 10.58 & 3.13 & 6.11 & 1.84 & $109.4 * * *$ & .000 & .43 \\
\hline F3 Partner lack of affection & 8.58 & 3.61 & 8.19 & 2.83 & 0.05 & .822 & .00 \\
\hline F4 Sexual passivity/control & 10.78 & 4.39 & 9.80 & 3.27 & 1.29 & .258 & .01 \\
\hline F5 Lack of erotic thoughts & 17.06 & 4.29 & 12.14 & 3.31 & $44.6^{* * * *}$ & .000 & .23 \\
\hline F6 Low self body-image & 6.67 & 2.54 & 6.32 & 2.58 & 0.55 & .461 & .00 \\
\hline Total & 66.97 & 12.70 & 52.53 & 10.33 & $36.90 * * *$ & .000 & .20 \\
\hline
\end{tabular}

$* p<.05 ; * * p<.01 ; * * * p<.001$

were used as discriminant variables, and sexual functioning as the criterion variable. The discriminant analysis produced a statistically significant function $\left(\chi^{2}(6)=51.41, p<.001\right)$, confirming that thoughts presented during sexual activity significantly differentiate women with and without sexual difficulties. A canonical correlation coefficient of $r=.72$ between the linear combination of the discriminant variables and the criterion variable showed the strong discriminating power of the discriminant function. The analysis of the individual contribution of each automatic thought dimension in the discrimination of clinical and control groups indicated that three of the thought dimensions show correlations higher than .30 with the discriminant function (see Table 3). Specifically, failure and disengagement thoughts $(r=.91)$, followed by lack of erotic thoughts $(r=.59)$, and sexual abuse thoughts $(r=.42)$, constituted the best predictors of group membership (clinical group versus control group). On the other hand, passivity and control thoughts, thoughts about partner's lack of affection, and low self body image thoughts showed a weak discriminant power.

The means of both groups in the discriminant function (clinical group $=1.07$, control group $=-.99$ ) indicated the higher negativity of the thought content presented by women with sexual dysfunction (positive positioning in a function characterized by negative thoughts). The identified discriminant function correctly classified $86.7 \%$ of the cases $(77.8 \%$ in the clinical group and $94.9 \%$ in the control group).

Table 3 Correlations between the female automatic thought dimensions and the discriminant function scores $(n=100)$

Note: Variables ordered by size of correlation within function

\begin{tabular}{ll}
\hline & Discriminant function \\
\hline F2 Failure/disengagement thoughts & .905 \\
F5 Lack of erotic thoughts & .589 \\
F1 Sexual abuse thoughts & .417 \\
F4 Sexual passivity and control & .126 \\
F3 Partner's lack of affection & .071 \\
F6 Low self body-image thoughts & .063 \\
\hline
\end{tabular}


Automatic Thoughts and Sexual Dysfunction in Men

Regarding the male sample, a multivariate analysis of covariance (MANCOVA) was performed using sexual functioning ( $1=$ clinical group, $2=$ control group $)$ as the independent variable, and the scores on the five dimensions of the automatic thought sub-scale as dependent variables. The variables age, marital status and educational level showed a statistically significant effect on the thoughts presented during sexual activity [age: $F(255,1106)=1.43$, $p<.001$; marital status: $F(25,1012)=1.62, p<.05$; educational level: $F(30,1078)=5.76$, $p<.001]$, and were included in the analysis as covariates (in order to control their effects). The multivariate analysis for sexual functioning (clinical group/control group), after controlled the effect of the demographic variables, was statistically significant $[F(5,212)=20.91$, $p<.001]$.

Results from the univariate analysis (see Table 4) confirmed the association between thoughts presented during sexual activity and sexual functioning. Men from the clinical group presented significantly more failure anticipation thoughts $(p<.001)$, erection concern thoughts $(p<.001)$, age and body related thoughts $(p<.01)$, and lack of erotic thoughts $(p<.001)$, compared to sexually healthy men. Scores on the total AT sub-scale were also significantly higher among men with sexual dysfunction $(p<.001)$. These differences assumed higher effect sizes regarding the erection concern thoughts $\left(\eta^{2}=.19\right)$ and failure anticipation thoughts $\left(\eta^{2}=.13\right)$.

A discriminant analysis was performed to investigate the individual contribution of each thought dimension. The five dimensions of the automatic thought sub-scale (matched for age, marital status and educational level) ${ }^{2}$ were used as discriminant variables, and sexual functioning as the criterion variable. The discriminant analysis produced a statistically significant function $\left(\chi^{2}(5)=49.06, p<.001\right)$ confirming that the automatic thought dimensions differentiate men from the clinical and control groups. A canonical correlation coefficient of $r=.70$ between the linear combination of the discriminant variables and the criterion variable, confirmed the discriminating power of the function. The analysis of the individual contribution of the thought dimensions showed that erection concern thoughts $(r=.61)$, followed by failure anticipation thoughts $(r=.47)$, and lack of erotic thoughts $(r=.42)$ were the most powerful discriminants. On the other hand, negative thoughts toward sexuality $(r=-.21)$ and age and body related thoughts $(r=.18)$, presented a weak discriminant power (see Table 5).

The means of both groups in the discriminant function indicated that, whereas men with sexual dysfunction had a positive mean score (0.89), sexually healthy individuals had a negative mean score $(-1.06)$, indicating the higher negativity of the thought content presented by men with sexual dysfunction (positive positioning in a function characterized by negative thoughts).

The identified discriminant function correctly classified $84.4 \%$ of the cases $(85.7 \%$ in the clinical group and $82.9 \%$ in the control group).

\footnotetext{
${ }^{2}$ In order to control the effect of the variables: age, marital status and educational level the discriminant analysis was performed using a sample constituted by the male clinical group $(n=49)$ and a sub-sample of the control group $(n=50)$ matched for age [clinical group $(M=42.98)$, control group $(M=42.46), t(93)=0.18, p=.86$ ], marital status $\left[\chi^{2}(3)=5.13, p=.16\right]$ and educational level [Mann-Whitney test for the comparison of the mean orders of the clinical (49.15) and control group (50.83), $z=-.30, p=.76]$.
} 
Table 4 Male thoughts as a function of sexual functioning (clinical group/control group): univariate analysis of covariance (covariables: age, marital status, and educational level)

\begin{tabular}{|c|c|c|c|c|c|c|c|}
\hline \multirow[t]{3}{*}{ Thought dimensions } & \multicolumn{4}{|c|}{ Groupsd } & \multirow[t]{3}{*}{$F(1,216)$} & \multirow[t]{3}{*}{$p$} & \multirow[t]{3}{*}{$\eta^{2}$} \\
\hline & \multicolumn{2}{|c|}{ Clinical $(n=40)$} & \multicolumn{2}{|c|}{ Control $(n=181)$} & & & \\
\hline & $M$ & SD & $M$ & SD & & & \\
\hline F1 Failure anticipation & 16.35 & 6.45 & 10.63 & 3.36 & $31.3 * * *$ & .000 & .13 \\
\hline F2 Erection concern thoughts & 20.38 & 8.02 & 10.76 & 4.12 & $51.9 * * *$ & .000 & .19 \\
\hline F3 Age and body thoughts & 8.45 & 3.55 & 5.93 & 2.24 & $9.1 * *$ & .003 & .04 \\
\hline F4 Negative sexual thoughts & 7.53 & 2.74 & 7.07 & 2.35 & 0.1 & .785 & .00 \\
\hline F5 Lack of erotic thoughts & 14.85 & 3.61 & 10.85 & 3.05 & $20.8 * * *$ & .000 & .09 \\
\hline Total & 67.55 & 17.77 & 45.24 & 9.69 & $49.5 * * *$ & .000 & .19 \\
\hline
\end{tabular}

$* p<.05 ; * * p<.01 ; * * * p<.001$

Table 5 Correlations between the male automatic thought dimensions and the discriminant function scores $(N=99)$

Note: Variables ordered by size of correlation within function

\begin{tabular}{ll}
\hline & Discriminant function \\
\hline F2 Erection concern thoughts & .605 \\
F1 Failure anticipation thoughts & .470 \\
F5 Lack of erotic thoughts & .421 \\
F4 Negative thoughts toward sex & -.214 \\
F3 Age and body related thoughts & .182 \\
\hline
\end{tabular}

\section{Discussion}

Data from the studies on cognitive content during sexual activity showed a clear distinction between individuals with and without sexual difficulties. In fact, results indicated that men and women with sexual dysfunction reported having had significantly more negative thoughts during sexual activity compared to sexually healthy individuals.

Regarding women, the strongest differences were observed for failure and disengagement thoughts, lack of erotic thoughts and sexual abuse thoughts. Women with sexual dysfunction reported having had significantly more thoughts from this nature relative to sexually healthy women. These findings were confirmed by a discriminant analysis that attributed to these three thought domains an elevated ability to correctly distinguish women with and without sexual difficulties. The most common examples from these thought domains were: "I'm not satisfying my partner", "I'm not getting turned on", "when will this be over?" (failure and disengagement thoughts), "the way he is talking turns me on", "these movements and positions are fabulous", "I'm the happiest woman on earth" (erotic thoughts), "this is disgusting", "how can I get out of this situation", "if I refuse to have sex, he will cheat on me", "he only wants to satisfy himself" (sexual abuse thoughts). Overall, these findings corroborate some clinical suggestions (Hawton 1985, 1989) and experimental findings (Adams et al. 1985; Elliot and O'Donohue 1997) indicating that cognitive distraction from erotic cues is strongly associated with female sexual dysfunction. Moreover, the content of that distraction seems to be partially related to sexual performance thoughts as suggested by Dove and Weiderman (2000). 
On the other hand, sexual passivity and control thoughts, lack of partner's affection, and low self-body image thoughts did not significantly discriminate between women with and without sexual dysfunction.

With respect to the male sample, results indicated that men with sexual dysfunction reported having had significantly more erection concern thoughts, failure anticipation, and lack of erotic thoughts, during sexual activity. These findings corroborate clinical suggestions (Carey et al. 1993; Hawton 1985; Rosen et al. 1994; Wincze and Barlow 1997; Zilbergeld 1999) as well as Barlow's model (1986), indicating that during sexual activity, sexually healthy men focus their attention on erotic cues, whereas men with sexual difficulties focus attention on anticipating failure and its catastrophic consequences: "this is not going anywhere", "I'm condemned to failure", "she will replace me with another guy', "it would be better to die than to be like this"; or on thoughts concerning erection demands: "I must be able to have intercourse", "I must achieve an erection", "Why isn't this working?".

On the other hand, age and body related thoughts and negative thoughts toward sexuality do not seem to differentiate clinical and control groups. Although men with sexual problems tend to focus their attention on aging issues ("I'm getting old", "it will never be the same again"), this domain did not contribute decisively to distinguish the clinical from the control group. With respect to negative thoughts toward sexuality, and contrary to predictions, no significantly different scores were found for men with and without sexual dysfunction. Thoughts like: "this is disgusting" or "this way of having sex is immoral" do not seem to be related to male sexual dysfunction.

Overall, these findings draw attention to thought patterns typical of both men and women with sexual difficulties. Although the current study did not allow comparisons between genders (since it is based on different instruments for men and women), results seemed to support the existence of a common underlying thought pattern in both sexes. Men and women with sexual dysfunction reported having had lack of erotic thoughts during sexual activity. Also, both anticipate sexual failure, which in the women's case seems to be associated with disengagement thoughts and in the men's case with thoughts about negative consequences.

Results from the current study should however be interpreted with caution due to several limitations. One main limitation regards the use of a sample partially employed in the validation studies of the SMQ (Nobre and Pinto-Gouveia 2003). The clinical sample of the present study was also used to assess the discriminant validity of the SMQ. Additional replications of the study using different samples should be conducted in order to test its findings. Another possible limitation was the heterogeneity of the clinical sample. In fact, men and women from the clinical sample presented with different sexual complaints, and it is possible that there is some specificity in the thought content according to different clinical diagnoses (research examining this potential specificity is undergoing at our site).

The fact that we did not control for the effect of psychopathology (e.g., depression, anxiety) is also a limitation, since clinical data suggest higher prevalence of depression and anxiety disorders in individuals with sexual dysfunction. Moreover, the fact that the administration conditions of the questionnaires were different for the clinical (administered at the clinic) and the control groups (administered at home) may also have exerted an effect on the findings.

Another important issue was the way automatic thoughts were assessed in the study. In fact we used a self-report measure asking participants to indicate the frequency in which a predetermined list of thoughts is likely to occur during sexual activity. Automatic thoughts are believed to occur automatically and mostly out of the individual's conscious awareness. Therefore, the use of a retrospective self-report measure may have biased the findings. Moreover, automatic thoughts are idiosyncratic in nature and presenting a predefined list of thoughts restricted the range of possible responses. 
Regardless of these limitations, we think that findings reinforce the importance played by cognitive phenomena, particularly the thought pattern presented during sexual activity, on sexual dysfunctional processes. Moreover, additional studies addressing the relationship between automatic thoughts, emotions and sexual response (Nobre 2003; Nobre and PintoGouveia, in press), as well as the way they are linked to core cognitive factors such as schemas (Nobre 2003; Nobre and Pinto-Gouveia 2007) and sexual beliefs (Nobre and Pinto-Gouveia 2006; Nobre, Pinto-Gouveia, and Gomes 2003) suggest the existence of a cognitive-emotional vulnerability to sexual dysfunctions (Nobre, in press). Overall, findings indicate important clinical implications. Individuals with sexual difficulties usually present significantly different cognitive patterns during sexual activity compared to sexually healthy individuals. These differences suggest potential benefits from increasing the use of cognitive techniques to the treatment of sexual dysfunction.

\section{References}

Abrahamson, D. J., Barlow, D. H., \& Abrahamson, L. S. (1989). Differential effects of performance demand and distraction on sexually functional and dysfunctional males. Journal of Abnormal Psychology, 98, 241-247.

Abrahamson, D. J., Barlow, D. H., Sakheim, D. K., Beck, J. G., \& Athanasiou, R. (1985). Effect of distraction on sexual responding in functional and dysfunctional men. Behavior Therapy, 16, 503-515.

Adams, A. E., Haynes, S. N., \& Brayer, M. A. (1985). Cognitive distraction in female sexual arousal. Psychohysiology, 22, 689-696.

American Psychiatric Association. (1994). Diagnostic and statistical manual of mental disorders (4th ed.). Washington, DC: Author.

Barlow, D. H. (1986). Causes of sexual dysfunction: The role of anxiety and cognitive interference. Journal of Consulting and Clinical Psychology, 54, 140-148.

Beck, A. T. (1967). Depression: Causes and treatment. Philadelphia: University of Pennsylvania Press.

Beck, A. T. (1996). Beyond belief: A theory of modes, personality, and psychopathology. In P. Salkovskis (Ed.), Frontiers of cognitive therapy. New York: Guilford Press.

Beck, J. S. (1995). Cognitive therapy: Basics and beyond. New York: Guilford Press.

Beck, J. G., Barlow, D. H., Sakheim, D. K., \& Abrahamson, D. J. (1987). Shock threat and sexual arousal: The role of selective attention, thought content, and affective states. Psychophysiology, 24, 165-172.

Cappelleri, J. C., Rosen, R. C., Smith, M. D., Mishra, A., \& Osterloh, I. H. (1999). Diagnostic evaluation of the erectile function domain of the International Index of Erectile Function. Urology, 54, 346-351.

Carey, M. P., Wincze, J. P., \& Meisler, A. W. (1993). Sexual dysfunction: Male erectile disorder. In D. H. Barlow (Eds.), Clinical handbook of psychological disorders: A step by step treatment manual (2nd ed., pp. 442-480). New York: Guilford Press.

Dove, N. L., \& Weiderman, M. W. (2000). Cognitive distraction and women's sexual functioning. Journal of Sex and Marital Therapy, 26, 67-78.

Elliot, A. N., \& O'Donohue, W. T. (1997). The effects of anxiety and distraction on sexual arousal in a nonclinical sample of heterosexual women. Archives of Sexual Behavior, 26, 607-624.

Farkas, G. M., Sine, L. F., \& Evans, I. M. (1979). The effects of distraction, performance demand, stimulus explicitness, and personality on objective and subjective measures of male sexual arousal. Behaviour Research and Therapy, 17, 25-32.

Geer, J. H., Fuhr, R. (1976). Cognitive factors in sexual arousal: The role of distraction. Journal of Consulting and Clinical Psychology, 4, 238-243.

Hawton, K. (1985). Sex therapy: A pratical guide. Northvale, NJ: Aronson.

Hawton, K. (1989). Sexual dysfunctions. In K. Hawton, P. M. Salkovskis, J. Kirk, \& D. M. Clark (Eds.), Cognitive behaviour therapy for psychiatric problems: A pratical guide. Oxford: Oxford University Press.

Nobre, P. J. (2003). Sexual dysfunction: contributions for the construction of a comprehensive model based on cognitive theory. Unpublished doctoral dissertation. Faculdade de Psicologia e Ciências da Educação da Universidade de Coimbra, Coimbra, Portugal.

Nobre, P. J. (in press). Determinants of sexual dysfunction: A cognitive-emotional model. Sexual and Relationship Therapy. 
Nobre, P. J., \& Pinto-Gouveia, J. (2003). Sexual modes questionnaire: Measure to assess the interaction between cognitions, emotions and sexual response. Journal of Sex Research, 40, 368-382.

Nobre, P. J., \& Pinto-Gouveia, J. (2006). Dysfunctional sexual beliefs as vulnerability factors to sexual dysfunction. Journal of Sex Research, 43, 68-75.

Nobre, P. J., \& Pinto-Gouveia, J. (2007). Cognitive schemas activated in negative sexual events and sexual dysfunction. Manuscript submitted for publication.

Nobre, P. J., \& Pinto-Gouveia, J. (in press). Cognitions, emotions and sexual response: Analysis of the interaction between automatic thoughts and emotional responses during sexual activity and its impact on sexual arousal. Journal of Sex Research.

Nobre, P. J., Pinto-Gouveia, J., \& Gomes, F. A. (2003). Sexual dysfunctional beliefs questionnaire: An instrument to assess sexual dysfunctional beliefs as vulnerability factors to sexual problems. Sexual and Relationship Therapy, 18, 101-135.

Rosen, R. C., Brown, C., Heiman, J., Leiblum, S., Meston, C., Shabsig, R. et al. (2000). The Female Sexual Function Index (FSFI): A multidimensional self-report instrument for the assessment of female sexual function. Journal of Sex and Marital Therapy, 26, 191-208.

Rosen, R. C., Leiblum, S. R., \& Spector, I. (1994). Psychologically based treatment for male erectile disorder: A cognitive-interpersonal model. Journal of Sex and Marital Therapy, 20, 67-85.

Rosen, R. C., Riley, A., Wagner, G., Osterloh, I. H., Kirkpatrck, J., \& Mishra, A. (1997). The International Index of Erectile Function (IIEF): A multidimensional scale for assessment of erectile dysfunction. Urology, 49, $822-830$.

Viglietta, M. B. (1982). The effects of anxiety versus distraction on sexual arousal in males. Unpublished doctoral dissertation, State University of New York at Albany, Albany, New York.

Wincze, J. P., \& Barlow, D. H. (1997). Enhancing sexuality: A problem solving approach (therapist guide). Albany, NY: Graywind.

Zilbergeld, B. (1999). The new male sexuality (rev. ed.). New York: Bantam. 